\title{
Few-body reactions at low energies
}

\author{
Henry R. Weller* \\ Duke University and TUNL \\ E-mail: weller@tunl.duke.edu
}

Mohammad W. Ahmed

Duke University and TUNL

E-mail: mohammad.ahmed@duke.edu

\begin{abstract}
The HI $\gamma \mathrm{S}$ facility is making it possible to perform studies of few body systems at a new level of accuracy and precision. A study of the photodisintegration of the deuteron using 100\% linearly polarized beams at 14 and $16 \mathrm{MeV}$ has determined the splittings of the three p-wave amplitudes involved in this process for the first time. These results show that the relativistic contributions, which when included in the theory lead to a positive value of the GDH integrand above $8 \mathrm{MeV}$, are valid. The same results are also used to extract the spin-polarizability of the deuteron for the first time. The experimental value is in good agreement with a recent effective field theory calculation.
\end{abstract}

6th International Workshop on Chiral Dynamics

July 6-10 2009

Bern, Switzerland

${ }^{*}$ Speaker. 


\section{Introduction}

The High Intensity $\gamma$-ray Source (HI $\gamma \mathrm{S})$ utilizes intra-cavity back-scattering of FEL light in order to produce intense $\gamma$-ray beams. A recently completed upgrade which included the installation of a $1.2 \mathrm{GeV}$ booster-injector has made it possible to produce nearly $100 \%$ linearly and circularly polarized beams having total intensities in excess of $10^{8} \gamma / \mathrm{s}$. Figure 1 displays the present layout of the $\mathrm{HI} \gamma \mathrm{S}$ facility, showing the linac, the new booster synchrotron, the upgraded RF system, the $1.2 \mathrm{GeV}$ storage ring, and the OK-4/OK-5 undulator system.

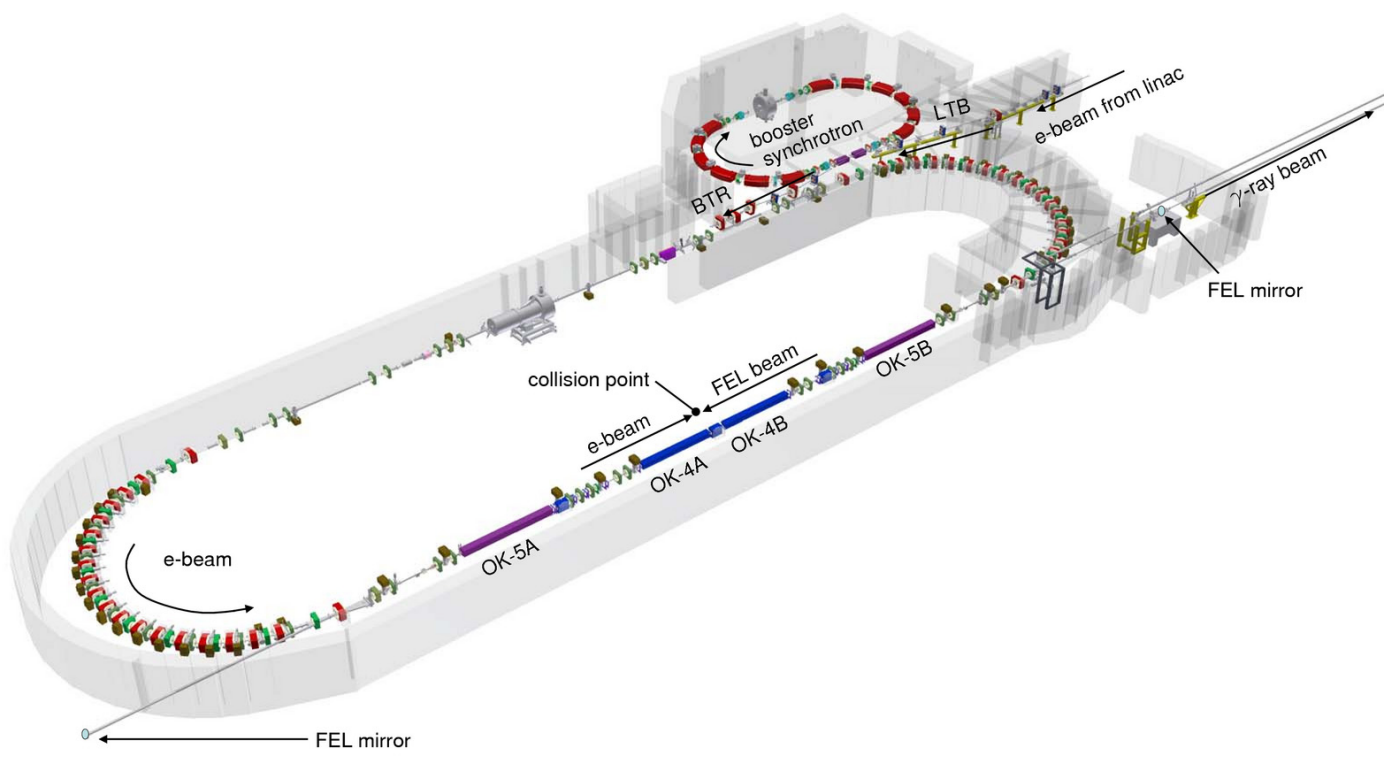

Figure 1: The fully operational $\mathrm{HI} \gamma \mathrm{S}$ facility in 2007. Since 2004, the following upgrade projects have been completed: (1) a newly commissioned 0.24 to $1.2 \mathrm{GeV}$ top-off booster synchrotron; (2) a new 34 meter long storage ring straight section for hosting a HOM-damped RF cavity and for implementing a new injection scheme with the booster; and (3) the newly commissioned OK-5 FEL in the second 34 m long FEL straight section.

A review of the facility and the overall research program can be found in Reference [1]. The energies of the $\mathrm{HI} \gamma \mathrm{S}$ beams, presently ranging from 2-to- $50 \mathrm{MeV}$, will soon be extended up to 100 $\mathrm{MeV}$. One of the major research programs which is being addressed with this facility is the study of few-body nuclear systems. This paper will present a brief review of recent results obtained in the case of photodisintegration of the deuteron. This example is intended to illustrate how the unique nearly mono-energetic beams at $\mathrm{HI} \gamma \mathrm{S}$ are making it possible to perform studies of few-body systems at a new level of accuracy and precision.

\section{Photodisintegration of the deuteron and p-wave splittings}

One of the experiments which has been performed using the HI $\gamma \mathrm{S}$ beams at relatively low energies was a study of the photodisintegration of the deuteron. This process was first discovered by Chadwick and Goldhaber [2] over 70 years ago using $2.62 \mathrm{MeV} \gamma$-rays from a Th $\mathrm{C}$ source. A year later H.A. Bethe and R. F. Bacher published a paper [3] which proved that this process at 

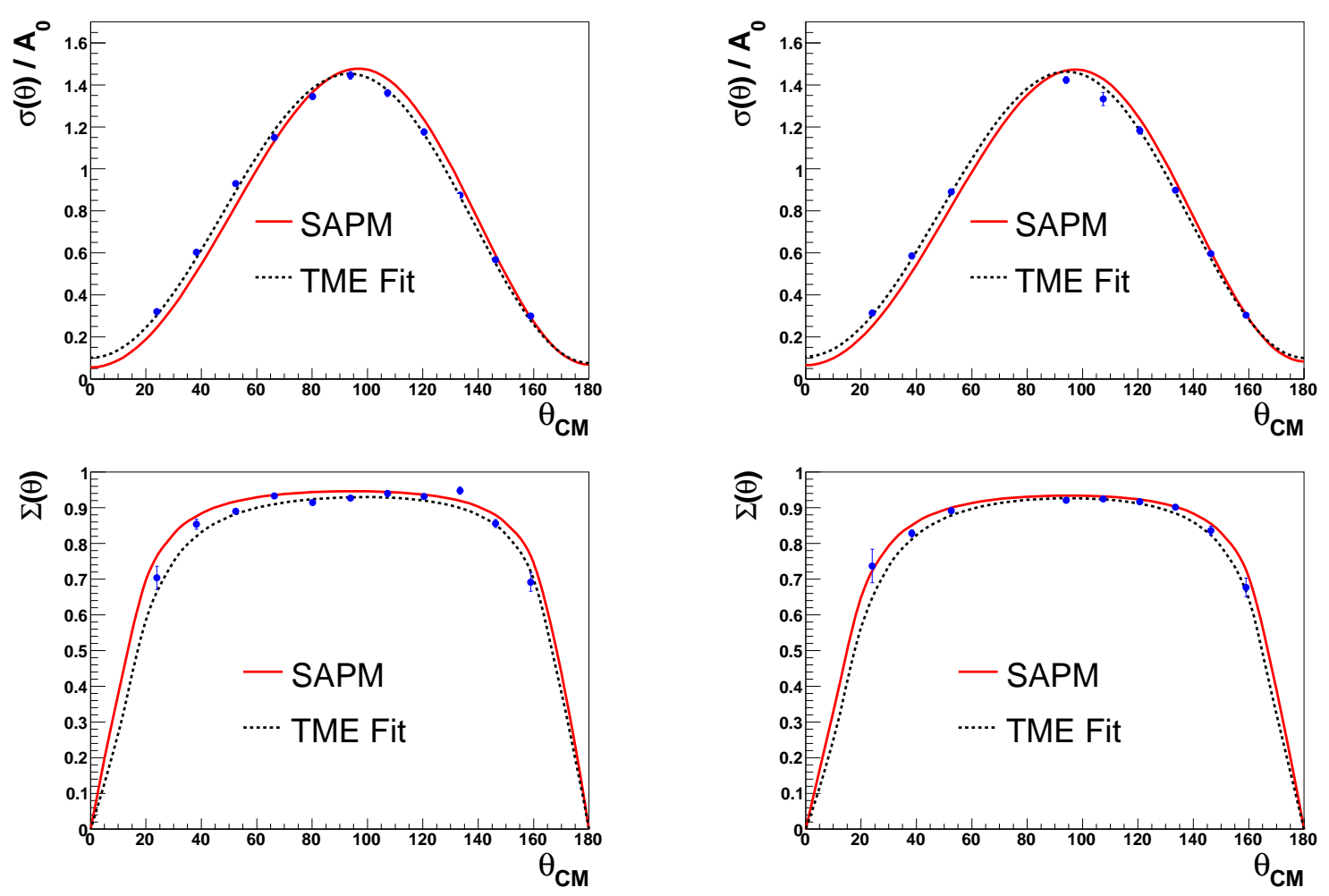

Figure 2: Cross section and analyzing power results at 14 (left) and $16 \mathrm{MeV}$ (right) along with fits from the extraction of the TME amplitudes. The solid red curves are from the calculation of SAPM and the dotted black curves represent the fit to the data. The errors on the data points are statistical only.

this and higher energies was dominated by electric dipole absorption (E1), and that the outgoing nucleons were therefore emitted in a state of relative angular momentum of 1-unit, ie. a p-wave.

Since the electric dipole operator is predominantly a $\Delta S=0$ operator, and since the ground state of the deuteron is mostly $\mathrm{S}=1, \ell=0$ and $\mathrm{J}=1$, there are actually $3 \mathrm{p}$-wave amplitudes, all of which have $S=1$ but with $J=0,1$ or 2 . We have known this for more than 70 years, but we have not had any information on the splittings of these three amplitudes-until now.

The experiment which took advantage of the unique qualities of the HI $\gamma \mathrm{S} \gamma$-ray beams to study this splitting employed a large neutron detector array (Blowfish) to perform a precision measurement of the $d(\vec{\gamma}, n) p$ reaction at 14 and $16 \mathrm{MeV}$.[4]

The Blowfish detector array consists of 88 BC-505 liquid scintillator cells located on the surface of a 16" diameter sphere centered on the target in 8 uniformly spaced arms of equal azimuthal angle $\phi$. The 11 cells in each arm are uniformly distributed between polar scattering angles $\theta$ of $22.5^{\circ}$ and $157.5^{\circ}$. The excellent pulse-shape discrimination (PSD) properties of BC-505 provide a strong handle on neutron $/ \gamma$ identification down to about $200 \mathrm{keV}_{e e}$. This particle ID capability is enhanced and extended to lower energies by taking advantage of the pulsed nature of the HI $\gamma \mathrm{S}$ $\gamma$-ray beam to provide time-of-flight (TOF) information. An LED based gain-monitoring system is used to track the gain of each detector during runs, thus allowing an accurate tracking of the hardware thresholds for each detector. [5] The broad coverage ( $25 \%$ of $4 \pi$ steradians) of the de- 
tector and its ability to be rotated about the beam axis makes it possible to achieve high statistical precision as well as an accurate determination of systematic effects.

The angular distribution data obtained using the Blowfish array at 14 and $16 \mathrm{MeV}$ were used to construct the unpolarized differential cross section, $\sigma(\theta)$, and the linear analyzing power, $\Sigma(\theta)$, which is simply the difference between the yields in and out of the plane of polarization, divided by the sum. A Monte Carlo simulation was developed using GEANT4 to correct for effects of multiple scattering and the finite geometry of the target and detectors. The results at 14 and $16 \mathrm{MeV}$ are shown in Fig. 2 where they are compared to the theoretical results of Schwamb and Arenhövel (SAPM) $[6,7,8]$. These observables can be written in terms of the amplitudes and phases of the reduced transition matrix elements (TMEs) which contribute to the reaction. Seven amplitudes were included in the fit corresponding to the (M1) ${ }^{1} \mathrm{~s}_{0},(\mathrm{E} 1)^{3} \mathrm{p}_{0},{ }^{3} \mathrm{p}_{1},{ }^{3} \mathrm{p}_{2}$, and $(\mathrm{E} 2)^{3} \mathrm{~d}_{1},{ }^{3} \mathrm{~d}_{2}$, and ${ }^{3} \mathrm{~d}_{3}$ terms. The relative phases were obtained from the $n-p$ scattering phase shifts (using the FermiWatson theorem). The results of this fitting procedure (see fits in Fig. 2) led to a splitting in the p-wave terms as shown in Fig. 3 for the $16 \mathrm{MeV}$ data set. The solid line at the value of 0.42 is the value which the squares of all three amplitudes would have if there were no splitting.

The fit indicated two solutions with identical $\chi^{2}$ values, which are shown in Fig 3 . This is the first determination of this p-wave splitting and, as seen in Fig. 3, is in excellent agreement (especially Solution 1) with the theoretical prediction. It is interesting to notice that in the case of the ${ }^{3} \mathrm{p}_{2}$ term our Solution 1 agress very well with the theoretical result if and only if the relativisitic contributions are included.

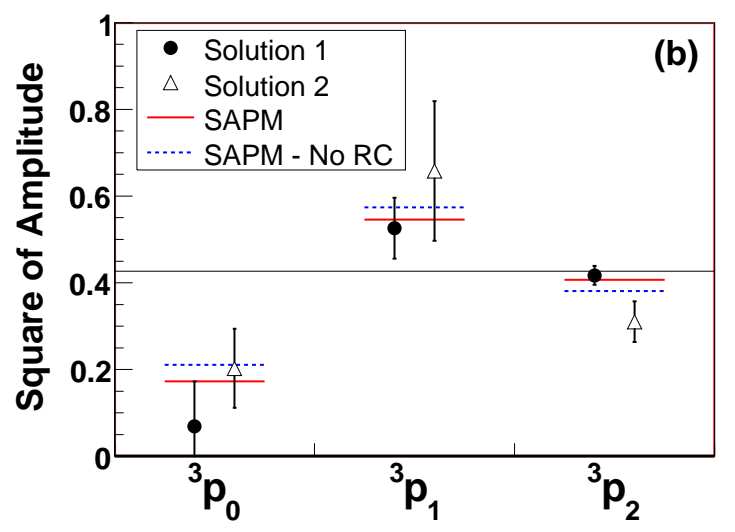

Figure 3: The squares of the three normalized triplet E1 p-wave amplitudes at $16 \mathrm{MeV}$ as extracted from the data and compared to the calculation of SAPM. The solid red line is the prediction from the full calculation and the dashed blue line is the prediction for the calculation without the inclusion of the relativistic contributions (RC). Error bars are statistical only.

\section{The Gerasimov-Drell-Hearn Sum Rule of the deuteron}

The Gerasimov-Drell-Hearn (GDH) sum rule connects the helicity structure of the photoabsorption cross section to the anomalous magnetic moment of the nuclear target. It is derived using Lorentz and gauge invariance, crossing symmetry, causality and unitarity of the forward 

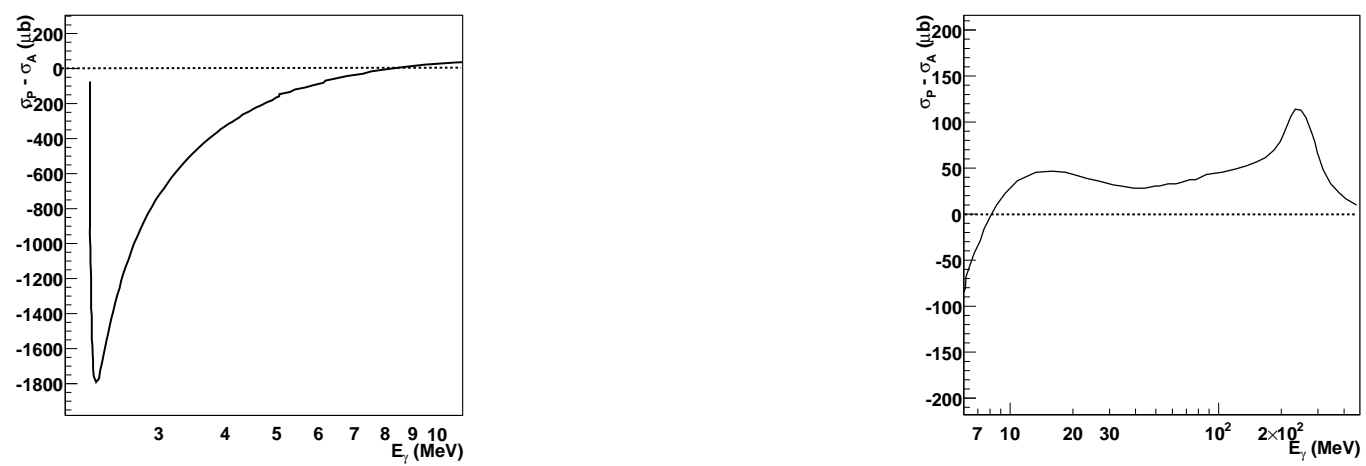

Figure 4: The GDH sum rule integrand $\left(\sigma_{P}-\sigma_{A}\right)$ for the deuteron as calculated by SAPM. The left figure shows the large negative near-threshold prediction, and the right figure shows the positive contributions which appear at higher energies when relativistic contributions are included.

Compton scattering amplitude, and is explicitly given by

$$
I_{T}=\int_{\omega_{\mathrm{th}}}^{\infty} d \omega \frac{\sigma_{P}(\omega)-\sigma_{A}(\omega)}{\omega}=4 \pi^{2} \alpha s_{T}\left(\frac{\kappa_{T}}{m_{T}}\right)^{2},
$$

where $\sigma_{P}$ and $\sigma_{A}$ are the cross sections for absorption of polarized photons of energy $\omega$ and helicities parallel and antiparallel to the target spin $s_{T}, \omega_{\mathrm{th}}$ is the threshold photon energy for inelastic processes, $\alpha$ is the fine-structure constant, and $m_{T}$ and $\kappa_{T}$ are the target mass and anomalous magnetic moment, respectively.

The recent interest in the nucleon and deuteron GDH sum rules stems from the study of the spin dependent structure functions in deep inelastic scattering. Since the proton and neutron have relatively large anomalous moments ( $\kappa_{p}=1.793$ and $\kappa_{n}=-1.913$, respectively), the corresponding values of $I$ obtained from Eq. (3.1) are large, $I_{p}=204.8 \mu \mathrm{b}$ and $I_{n}=232.5 \mu \mathrm{b}$, while the deuteron, for which $\kappa_{d}=-0.143$, has a comparatively small $I_{d}=0.652 \mu \mathrm{b}$. As has been previously discussed, one should expect to observe the sum of the proton and neutron strengths (and more) in the deuteron above pion threshold, indicating that a large negative contribution of about this size $(-436 \mu \mathrm{b})$ should exist below this threshold. Indeed, Arenhövel [8] and others point out that the photodisintegration channel, which is the only photo-absorption process below the pion threshold, gives a large negative contribution arising from the M1 transition to the resonant ${ }^{1} \mathrm{~S}_{0}$ state just above the deuteron breakup threshold $\left(\omega-\omega_{\mathrm{h}}<100 \mathrm{keV}\right)$, since this state can only be formed if the deuteron spin and photon helicity are antiparallel. The predictions of these calculations, shown in Fig. 4, indicate a large negative contribution just above threshold followed by a positive contribution which arises from including a relativistic contribution in the theory.

The GDH Collaboration (Spokespersons: M. W. Ahmed, B. Norum, R. Pywell, and H. R. Weller) is a HI $\gamma \mathrm{S}$ Collaboration which is dedicated to measuring the integrand of this sum rule. There are two main phases to this work. The first phase has recently been completed. It consisted of using the linearly polarized gamma-ray beams and unpolarized targets to measure the nearthreshold contribution to the GDH sum-rule integral of the deuteron. The results of this study have been published in Refs. [4, 9]. 
In order to see how this is possible, we note that the difference of the parallel and antiparallel cross sections $\left(\sigma_{P}-\sigma_{A}\right)$ can be expressed in terms of the Transition Matrix Elements (TMEs) of the reaction. In the region just above threshold it is known that only s-wave (M1) and p-wave (E1) TMEs contribute. We shall use the notation ${ }^{2 S+1} S_{J+1}$, and ${ }^{2 S+1} p_{J+1}$ to represent the TMEs (for outgoing neutrons having $l=0$ (s) or $l=1(\mathrm{p})$, respectively). In order to simplify the results, we set the ${ }^{1} \mathrm{p}_{1}$ term to zero, since it corresponds to spin-flip E1 and is expected to be negligibly small. The total photodisintegration cross section is given by:

$$
\begin{aligned}
\sigma_{\text {TOT }}= & \frac{\pi}{6 \kappa^{2}}\left[\left.\left.\right|^{3} p_{0}\right|^{2}+\left.\left.3\right|^{3} p_{1}\right|^{2}\right. \\
& \left.+\left.\left.5\right|^{3} p_{2}\right|^{2}+\left|{ }^{1} s_{0}\right|^{2}+\left.\left.3\right|^{3} s_{1}\right|^{2}\right],
\end{aligned}
$$

while $\sigma_{P}-\sigma_{A}$ is given by:

$$
\begin{aligned}
\Delta \sigma= & \sigma_{P}-\sigma_{A} \\
= & \frac{\pi}{2 k^{2}}\left[-\left.\left.\right|^{3} p_{0}\right|^{2}-\left.\left.\frac{3}{2}\right|^{3} p_{1}\right|^{2}\right. \\
& \left.+\frac{5}{2}\left|{ }^{5} p_{2}\right|^{2}-\left|{ }^{1} s_{0}\right|^{2}-\left.\left.\frac{3}{2}\right|^{3} s_{1}\right|^{2}\right],
\end{aligned}
$$

Note that if the three p-wave E1 TMEs are identical (i.e., no $j$ dependence), then $\sigma_{P}-\sigma_{A}$ is determined by the s-wave terms alone. Furthermore, if the $\beta^{\beta} s_{1} \mid$ term is negligible, as expected, then we can write:

$$
\begin{aligned}
\Delta \sigma & =\left.\left.\frac{\pi}{2 k^{2}}\right|^{1} s_{0}\right|^{2} \\
& =-3 \sigma(M 1) .
\end{aligned}
$$

Measurements of the analyzing powers at $90^{\circ}$ were performed at $\mathrm{HI} \gamma \mathrm{S}$ and used to determine the fractional M1 contribution S(M1) by assuming that the reaction proceeds via a mixture of M1(swave) and E1(p-wave) transitions [9]. These results were multiplied by the theoretical values of the total photodisintegration cross sections to produce the absolute M1 cross section as a function of energy which were converted to the values of $\sigma_{P}-\sigma_{A}$ using Eq. 3.4. Additional results can be obtained from previously measured polarized neutron capture on proton analyzing powers as well as from unpolarized photodisintegration data in which the outgoing neutron polarization was measured. In this case the analyzing power at $90^{\circ}$ arises from the interference of the s- and p-wave amplitudes and is proportional to the phase difference between them:

$$
A_{y}\left(90^{\circ}\right)=\frac{3|S||P| \sin \left(\phi_{S}-\phi_{P}\right)}{1+\frac{9}{2} P^{2}},
$$

where

$$
9|P|^{2}+|S|^{2}=1.0
$$

Since the phase difference can be obtained using the n-p elastic scattering phase shifts, the measured values of $\mathrm{A}_{y}\left(90^{\circ}\right)$ determine the percentage of the cross section due to s-waves (which is the 


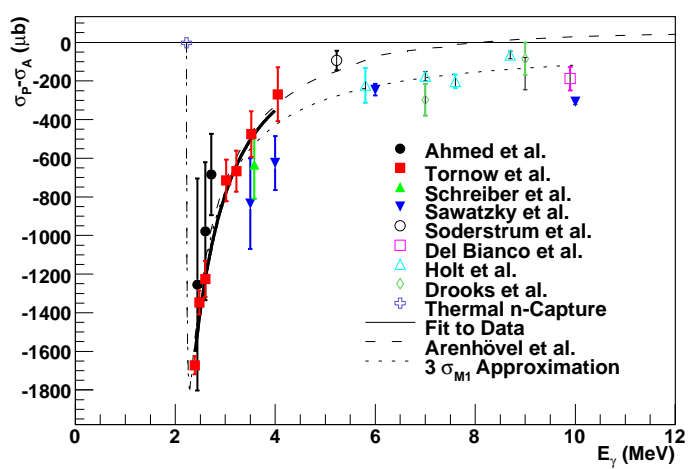

Figure 5: Experimental data points for the GDH sum rule integrand are shown along with their statistical errors. The thick solid curve is a fit to the data while the dashed curve is a prediction from [8]. The dotted curve is the same theory assuming $\sigma_{P}-\sigma_{A}=-3 \sigma(\mathrm{M} 1)$. This figure is taken from Ref. [9].

M1 part). Results from these data are included in Fig. 5, along with the theoretical predictions of [8]. The basic assumption made in obtaining these results is that the photodisintegration of the deuteron at these energies can be described using M1 (with outgoing s-waves), E1 (with outgoing p-waves) and E2 (with outgoing d-waves) TMEs. We have also assumed that there is no splitting either in the triplet $\mathrm{p}$-waves or in the triplet d-waves. This assumption means that the $\mathrm{p}$-and d-wave contributions drop out of the GDH integrand.

The full theory shown in Fig. 5, when integrated from threshold up to $10 \mathrm{MeV}$ using Eq. 1, predicts a value of $-634 \mu \mathrm{b}$. The theory also predicts that the value of this integral up to pion threshold is $-520 \mu \mathrm{b}$. A positive contribution (theoretically arising from a relativistic contribution) at energies between $10 \mathrm{MeV}$ and pion-threshold is required to obtain this result. As will be seen below, the first experimental verification of the presence of this relativistic effect is contained in the p-wave splitting results described above.

As seen in Eq. 3.3, a knowledge of the p-wave splittings can be translated into a value of the GDH integrand. The quantity $\sigma_{P}-\sigma_{A}$ (Eq. 3.3) was calculated for both solutions with the results as shown in Fig. 6. Here we see that Solution 1 agrees very well with the theoretical prediction especially when relativistic contributions are included in the theory. This is the first experimental evidence for a positive value of the GDH integrand in this energy region, and supports the need to include relativistic contributions in the theory. In addition to providing a value of the GDH integrand at these energies, this method of evaluating the GDH integrand provides us with a deep insight into the origin of this positive value, a result which cannot be obtained by a direct measurement. These results have been published in $[4,9]$.

In addition to determining the GDH integral value in this near threshold energy region, these results can be used to determine the value of the so-called forward spin-polarizability $\not x$, which is given by:

$$
\gamma_{0}=\frac{-1}{8 \pi^{2}} \int_{\omega_{\mathrm{th}}}^{\infty} d \omega \frac{\sigma_{P}(\omega)-\sigma_{A}(\omega)}{\omega^{3}}
$$

The result of integrating from threshold to $6 \mathrm{MeV}$ is $\gamma_{0}=3.75_{-}^{+} 0.18 \mathrm{fm}^{4}$. This agrees with the result of an effective field theory calculation at leading order, which predicts a value of $3.762 \mathrm{fm}^{4}$, 


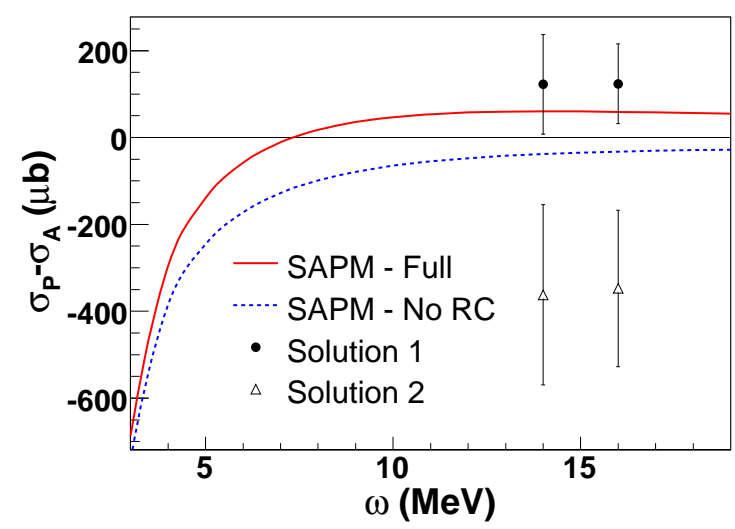

Figure 6: $\sigma_{P}-\sigma_{A}$ for the two solutions extracted for the TME amplitudes of the reaction at each beam energy. Also shown is the full calculation of SAPM and the calculation without relativistic contributions (RC). Error bars are statistical only.

but is somewhat lower than the next-to-leading order result of $4.262 \mathrm{fm}^{4}$ [10]. Although the integral in this case is quite rapidly converging, the very limited energy range of the present experimental results is almost certainly not converged. A full report of this first determination of the forward spin-polarizability of the deuteron can be found in Ref. [9].

Although these indirect measurements have been very instructive, the continuation of this method to higher energies is not practical since the number of contributing TMEs is known to grow. The number of unknowns will therefore be too great to be able to obtain meaningful solutions. For this reason we are proposing to make direct measurements of the GDH integrand for the deuteron at energies between 5 and $100 \mathrm{MeV}$ during the next three years.

The Blowfish detector, a frozen-spin polarized deuterium target [11], and circularly polarized gamma rays from the OK-5 helical undulator are required for a direct measurement of the GDH integrand. The frozen-spin target is under construction and is expected to be available in 2010.[11] The OK-5 system, which is a helical undulator based free-electron laser, has been installed and has produced circularly polarized gamma rays which were used in the first measurements of the GDH integrand for ${ }^{3} \mathrm{He}$ [12].

The GDH Collaboration anticipates starting this experiment in 2010. Detailed studies have indicated that a measurement of the integral over the energy range between 5 and $50 \mathrm{MeV}$ to an accuracy of $5 \%$ can be achieved in less than 300 hours of running with a beam intensity of 10 $\gamma_{\mathrm{s}} / \mathrm{s}$. Beams with energies up to $100 \mathrm{MeV}$ are expected to become available in late 2010. This will allow the Collaboration to extend the measurement of the GDH integrand up to $100 \mathrm{MeV}$ starting in 2011. Ultimately, the Collaboration will extend these measurements up to pion threshold, but this must await the development of mirrors needed to produce beams with energies up to $158 \mathrm{MeV}$.

\section{References}

[1] H. R. Weller et al., Prog. Part. Nucl. Phys, 62, 257-303 (2009)

[2] J. Chadwick and M. Goldhaber, Proc. Roy. Soc., A151, 479 (1935)

[3] H. A. Bethe and R. F. Bacher, Rev. Mod. Phys., 8, 82-229 (1936) 
[4] M. A. Blackston et al., Phys. Rev., C78, 034003 (2008)

[5] R. Pywell, University of Saskatchewan, private communication (2008)

[6] M. Schwamb and H. Arenhövel, Nucl. Phys., A690, 682 (2001)

[7] M. Schwamb and H. Arenhövel, Nucl. Phys., A696, 556 (2001)

[8] H. Arenhövel, A. Fix, and M. Schwamb, Phys. Rev. Lett., 93, 202301 (2004)

[9] M. W. Ahmed et al., Phys. Rev., C77, 044005 (2008)

[10] X. Ji and Y. Li, Phys. Lett., B591, 76 (2004)

[11] D. Crabb, University of Virginia, private communication (2009)

[12] H. Gao, Duke University, private communication (2009) 